\title{
Prevalence of Intestinal Obstruction in Patients Attending Tertiary Care Institute of Gujarat
}

\author{
Akhilesh G Sukhlecha ${ }^{1}$ \\ ${ }^{1}$ Associate Professor, Department of Surgery, Gujarat Adani Institute of Medical Science, Bhuj, Kutch, Gujarat.
}

\section{Abstract}

Background: Intestinal defined as obstruction in forward propulsion of the contents as obstruction in the intestine either due to mechanical or neurological cause. Aim of the present study was to know the various causes of intestinal obstruction and also to analyse the symptomatology of intestinal obstruction. Subjects and Methods: The present study was under taken with the aim to evaluate the various causes and the mode of presentation. A total of 60 cases of the intestinal obstruction attending Department of Surgery, tertiary care institute of Gujarat for the period of two years were included in the study. All the patients were assessed clinically in details after admission. All the pathological and biochemical investigations were carried out in all the patients. Results: The mean age was found to be 43 years. The intestinal obstruction was found to be more common in male as compared to female. More number of cases was of small bowl obstruction as compared to large bowel obstruction. Majority of the patients had the symptoms of tenderness. It was found in $93 \%$ of the cases. Next was the pain present in the abdomen found in $83 \%$, followed by vomiting present in $80 \%$ of the cases. Conclusion: The incidence of intestinal obstruction is more common in males compared to females. Patients with a clinical picture of obstruction of the bowel demand vigorous correction of fluid and electrolyte, which can be severe, and life threatening. Intestinal obstruction is more common in the age group of 30-60 years. Large bowel obstruction is more common in patients above 40 years than in younger group. Intestinal obstruction was also found more common in young children.

Keywords: children, electrolyte, intestinal obstruction, small bowl obstruction.

Corresponding Author: Dr Akhilesh G Sukhlecha, 28 new nirmalsinh wadi, near pramukhswami temple, Bhuj, Kutch, Gujarat, India-370001.

Received: May 2019

Accepted: June 2019

\section{Introduction}

Intestinal obstruction is a common surgical emergency all over the world. It is defined as obstruction in forward propulsion of the contents as obstruction in the intestine either due to mechanical or neurological cause. It is predisposed by varying underlying anomalies and diseases, which are difficult to define preoperatively. ${ }^{[1,2]}$

Though intestinal obstruction can be diagnosed easily, the underlying cause except postoperative adhesions and external hernias are difficult to be diagnosed preoperatively. ${ }^{[3]}$

Intestinal obstruction is a blockage that keeps food or liquid from passing through your small intestine or large intestine (colon). Causes of intestinal obstruction may include fibrous bands of tissue (adhesions) in the abdomen that form after surgery, an inflamed intestine (Crohn's disease), infected pouches in your intestine (diverticulitis), hernias and colon cancer. Without treatment, the blocked parts of the intestine can die, leading to serious problems. However, with prompt medical care, intestinal obstruction often can be successfully treated. ${ }^{[4,5]}$

Although the mortality due to acute intestinal obstruction is decreasing with better understanding of pathophysiology, improvement in diagnostic techniques, fluid and electrolyte, correction, much potent anti-microbials and the knowledge of intensive care. ${ }^{[6,7]}$

Various studies in India report about $8-12 \%$ in recent times. Most of the mortalities occurs in elderly individuals who seek late treatment and who are having associated preexisting comorbid conditions. ${ }^{[8]}$

The dictum of never let the sun set or rise in small bowel obstruction has made early surgical intervention for intestinal obstruction. This in turn has reduced the incidence of strangulation of bowel, which was major cause of mortality in already ill patient. ${ }^{[9]}$

Early diagnosis of obstruction skillful operative management, proper technique during surgery and intensive postoperative treatment carries a grateful result. ${ }^{[6]}$ Hence the aim of the present study was to know the various causes of intestinal obstruction and also to analyse the symptomatology of intestinal obstruction.

\section{Subjects and Methods}

In the routine practise every surgeons have to come across the surgical emergency of the intestinal obstruction. The treatment of such emergency depends on the early diagnosis and the rightful management. 
The present study was under taken with the aim to evaluate the various cause and the mode of presentation. A total of 60 cases of the intestinal obstruction Department of Surgery, tertiary care institute of Gujarat for the period of two years were included in the study. All the age group patients were included in the study. All the patients attended to the opd or the emergency department at the medical college \& hospital. All the patients had clinical picture of intestinal obstruction. Those who had hernia with pain, constipation and vomiting were included in the study.

All those who had dynamic or sub acute intestinal obstruction and treated conservatively were excluded from the study. All the patients were assessed clinically in details after admission. All the pathological and biochemical investigations were carried out in all the patients.

Patients who showed reduction in abdominal distension and improvement in general Condition, especially in individuals with postoperative adhesions a chance of conservative management was taken for further 12 to 24 hours, those who showed improvement by moving bowels, reduction in pain/tenderness in decided for conservative treatment, such individuals are excluded in this study.

Statistical Methods: Chi-square and Fisher Exact test has been used to find the significant of proportion of Postoperative complications in association with etiology of Intestinal Obstruction.

\section{Results}

A total of 60 cases of the intestinal obstructions were included in the study. The study period was of two years.

Table 1: Age distribution
\begin{tabular}{|l|l|}
\hline Age & Total Cases \\
\hline $0-10$ & 14 \\
\hline $11-20$ & 4 \\
\hline $21-30$ & 6 \\
\hline $31-40$ & 12 \\
\hline $41-50$ & 12 \\
\hline $51-60$ & 4 \\
\hline $61-70$ & 2 \\
\hline $71-80$ & 2 \\
\hline $81-90$ & 4 \\
\hline Total & 60 \\
\hline
\end{tabular}

Table 2: levels of obstruction

\begin{tabular}{|l|l|}
\hline Small bowel & Large bowel \\
\hline 40 & 20 \\
\hline
\end{tabular}

Table 3: Analyses of the sign and symptoms.

\begin{tabular}{|l|l|l|}
\hline Sr. No. & Signs \& Symptoms & Percentage (\%) \\
\hline 1 & Tenderness & 93 \\
\hline 2 & Abdominal pain & 83 \\
\hline 3 & Vomiting & 80 \\
\hline 4 & Distension & 70 \\
\hline 5 & Guarding & 45 \\
\hline 6 & Rigidity & 30 \\
\hline 7 & Constipation & 22 \\
\hline 8 & Palpable mass & 13 \\
\hline 9 & Groin swelling & 8 \\
\hline
\end{tabular}

The present study was done in all the age groups that range from new born to 90 years. The mean age was found to be 43 years. The intestinal obstruction was found to be more common in male as compared to female. The male to female ratio was found to be $2: 1$. More number of cases was of small bowl obstruction as compared to large bowel obstruction.

On analysis of the symptoms that were present and also used to diagnose the intestinal obstruction were as follows: Majority of the patients had the symptoms of tenderness. It was found in $93 \%$ of the cases. Next was the pain present in the abdomen found in $83 \%$, followed by vomiting present in $80 \%$ of the cases. The other signs and symptoms included were distension, guarding, rigidity, constipation, palpable mass and groin swelling.

\section{Discussion}

Intestinal obstruction is one of the commonly encountered clinical entities. There is probably not a day that goes by, in which a clinical surgeon does not atleast once, come across the possible diagnosis of intestinal obstruction Intestinal obstruction continues to be a frequent emergency, which surgeons have to face (1-4\% of emergency operations). ${ }^{[10]}$

Brewer et $\mathrm{al}^{[11]}$ analysed 1000 consecutive abdominal surgeries in 1976 and reported an incidence of $2.5 \%$. In our hospital the incidence of intestinal obstruction out of all the abdominal surgeries was about $2.2 \%$. The involvement of small bowel in obstruction is much more common than that of large bowel.

The delay in the treatment will lead to high mortality. Since the advancement in understanding the anatomy/physiology fluid and electrolyte management along with modern antibiotics and intensive care unit. The mortality has been decreasing consistently, associated medical problems and advanced age carries a considerable contribution in adding the mortality. ${ }^{[6]}$

The acute intestinal obstruction occurs in all age groups. The age distribution in our series ranges from newborn to 90 years with mean age of 43 years. Maximum incidence was seen between age group of 41-50 yrs (30\%) followed by the age group $0-10(23 \%)$.

The male preponderance is consistent with series reported from other part of world. Fuzan and Lee reported 2:1 male to female ratio. Same results were obtained in our study where we found males were affected more as compared to females.

\section{Conclusion}

From the stud, the following conclusion can be drawn: The occurrence of intestinal obstruction is more in small bowel. All age groups from newborn to elderly were involved. The incidence of intestinal obstruction is more common in males compared to females. Patients with a clinical picture of obstruction of the bowel demand vigorous correction of fluid and electrolyte, which can be severe, and life threatening. Intestinal obstruction is more common in the age group of 30-60 years. Large bowel obstruction is more common in patients above 40 years than in younger group. Intestinal obstruction was also found more common in young children (First decade).

\section{References}


1. Sridhara K: The Clinical Study and Management of Acute Intestinal Obstruction In Adults. 2011.

2. Spinn MP, Guha S: Small bowel tumors. Textbook of Clinical Gastroenterology and Hepatology 2012:421.

3. Stewart RM, Page CP, Brender J, Schwesinger W, Eisenhut D: The incidence and risk of early postoperative small bowel obstruction: a cohort study. The American journal of surgery 1987, 154:643-7.

4. Treves F: Intestinal Obstruction: Its Varieties, with Their Pathology, Diagnosis, and Treatment. The Jacksonian Prize Essay of the Royal College of Surgeons of England, 1883: HC Lea's Son \& Company, 1884.

5. Miller EM: Bowel obstruction in the new born. Annals of surgery 1939, 110:587.

6. Naveen N, Mukherjee A, Nataraj Y, LingeGowda S: A clinical study of intestinal obstruction and its surgical management in rural population. Hernia 2013, 10:20
7. Mukherjee A: Intestinal obstruction, adhesions, hernia, intraperitoneal malignancy, tubercular stricture. A CLINICAL STUDY OF INTESTINAL OBSTRUCTION AND ITS SURGICAL MANAGEMENT IN RURAL POPULATION 2013.

8. Kawai K, Gebremeskel BG, Acosta CJ: Systematic review of incidence and complications of herpes zoster: towards a global perspective. BMJ open 2014, 4:e004833.

9. Bansod A, Shamkuwar A: Study of large bowel obstruction in adults. Journal of Evolution of Medical and Dental Sciences 2013, 2:3439-50.

10. DRUCKER WR, Davis JH, Holden WD, REAGAN JR: Hemorrhagic Necrosis of the Intestine: A Clinical Syndrome: Presence Without Organic Vascular Occlusion. Archives of Surgery 1964, 89:42-53.

11. Brewer RJ, Golden GT, Hitch DC, Rudolf LE, Wangensteen SL: Abdominal pain: an analysis of 1,000 consecutive cases in a university hospital emergency room. The American journal of surgery 1976, 131:219-23.

Copyright: (C) the author(s), 2019. It is an open-access article distributed under the terms of the Creative Commons Attribution License (CC BY 4.0), which permits authors to retain ownership of the copyright for their content, and allow anyone to download, reuse, reprint, modify, distribute and/or copy the content as long as the original authors and source are cited.

How to cite this article: Sukhlecha AG. Prevalence of Intestinal Obstruction in Patients Attending Tertiary Care Institute of Gujarat. Acad. J Surg. 2019;2(1):10-12.

DOI: dx.doi.org/10.21276/ajs.2019.2.1.4

Source of Support: Nil, Conflict of Interest: None declared 